\title{
ELEMENTARY EQUIVALENCE AND THE COMMUTATOR SUBGROUP
}

\author{
by L. P. D. VAN DEN DRIES, A. M. W. GLASS, ANGUS MACINTYRE, \\ ALAN H. MEKLER, and JOHN POLAND ${ }^{1}$
}

(Received 23 September, 1980)

If $G$ and $H$ are elementarily equivalent groups (that is, no elementary statement of group theory distinguishes between $G$ and $H$ ) then the definable subgroups of $G$ are elementarily equivalent to the corresponding ones of $H$. But the commutator subgroup $G^{\prime}$ of $G$, consisting of all products of commutators $[a, b]=a^{-1} b^{-1} a b$ of elements $a$ and $b$ of $G$, may not be definable. Must $G^{\prime}$ and $H^{\prime}$ be elementarily equivalent?

A first thought might be that if $G^{\prime}$ is not definable, even allowing parameters, then it will follow that there is $H \equiv G$ with $H^{\prime} \not \equiv G^{\prime}$. However, this naive idea fails.

EXAMPLE. Let $G$ be the direct product of a countably-infinite free-nilpotent-of-class-2and-exponent-3 group with a countably-infinite group of exponent 3 . If $H \equiv G$, then $H^{\prime}$ and $G^{\prime}$ are infinite abelian groups of exponent 3 and hence $H^{\prime} \equiv G^{\prime}$. But $G^{\prime}$ is not definable (by an obvious modification of the lemma below).

Despite this example, we will show the following theorem.

Theorem. There exists groups $G$ and $H$ such that $G \equiv H$ (in fact $G<H$ ) but $G^{\prime} \not \equiv H^{\prime}$.

If $G \equiv H$, then no quantifier-free sentence will distinguish between $G^{\prime}$ and $H^{\prime}$, and the same is true for an $\forall$-sentence (and hence an $\exists$-sentence). About the simplest sentence to consider next would be something of the form $\forall x \exists y\left(x=y^{2}\right)$, so our construction begins with a group $F$ of nilpotent class 2 and reduces modulo a normal subgroup that makes each commutator a square, but there is no uniform bound on the length of the commutator word being squared. An ultrapower of the resulting group $G$ produces $H$ with some non-squares in its commutator subgroup. Now for the details. We will say that $G$ is a nil-2 group if it is a group satisfying $\forall x \forall y \forall z[x, y, z]=1$, where $[x, y, z]=[[x, y], z]$.

LEMMA. There is a nil-2 group $G$ with the following pair of properties:

(a) every element of $G^{\prime}$ is the square of an element of $G^{\prime}$,

(b) for every $n<\omega$, there exist $g_{n}$ and $h_{n}$ in $G$ such that $\left[g_{n}, h_{n}\right]$ is not the square of any product of at most $n$ commutators.

Proof of the lemma. Our group $G$ will be $F / K$ where $F$ is the free nil-2 group on $\left\{a_{n}: n<\omega\right\}$ and $K$ will be a central subgroup generated by relations $R_{n}(n<\omega)$. For any word $w$ in $F^{\prime}$, let $l(w)$ be the minimum number of commutators required to witness that $w$ is in $F^{\prime}$ and note that since $F$ is free nil-2 on $\left\{a_{n}: n<\omega\right\}$ then $[a, b c]=[a, b][a, c]$ and $F^{\prime}$ is

1 The authors would like to thank Bowling Green State University, Talisker Malt, and the Central States Universities, Inc., for making these results possible. Research was supported in part by a number of granting agencies.

Glasgow Math. J. 23 (1982) 115-117. 
a free abelian group with basis $\left\{\left[a_{i}, a_{i}\right]: i<j<\omega\right\}$ (see [1]). Hence for each $m<\omega$ there exists $w$ in $F^{\prime}$ (for example $\left.w=\left[a_{0}, a_{1}\right]\left[a_{2}, a_{3}\right] \ldots\left[a_{2 m}, a_{2 m+1}\right]\right)$ such that $l\left(w^{t}\right) \geq m$ for all integers $t \neq 0$. For, suppose $\left(\left[a_{0}, a_{1}\right] \ldots\left[a_{2 m}, a_{2 m+1}\right]\right)^{t}$ were the product of fewer than $m$ commutators for some $t \neq 0$. As $F^{\prime}$ is free abelian on $\left\{\left[a_{p}, a_{q}\right]: p<q<\omega\right\}$, we have for some integers $x(i, j)$ and $y(i, j)$

where

$$
\begin{aligned}
\prod_{0 \leq p \leq m}\left[a_{2 p}, a_{2 p+1}\right]^{\mathrm{j}} & =\prod_{0 \leq j<m-1}\left[\prod_{i \leq 2 m+1} a_{i}^{x(i, j)}, \prod_{i \leq 2 m+1} a_{i}^{y(i, j)}\right] \\
& =\prod_{0 \leq p<q \leq 2 m+1}\left[a_{p}, a_{q}\right]^{v(p . q)},
\end{aligned}
$$

$$
v(p, q)=\sum_{0 \leq i<m-1}\{x(p, j) y(q, j)-x(q, j) y(p, j)\}
$$

Hence the number

$$
v(p, q)=\sum_{0 \leq i<m-1}\{x(p, j) y(q, j)-x(q, j) y(p, j)\}
$$

equals $t$ if $p$ is even and $p+1=q$ and is 0 otherwise. If we let

$$
A=\left[\begin{array}{ccccc}
x(0,0) & -y(0,0) & \ldots & x(0, m-2) & -y(0, m-2) \\
\cdot & \cdot & \ldots & \cdot & \cdot \\
x(2 m+1,0) & -y(2 m+1,0) & \ldots & x(2 m+1, m-2) & -y(2 m+1, m-2)
\end{array}\right]_{(2 m+2) \times(2 m-2)}
$$

and

$$
B=\left[\begin{array}{lllll}
y(1,0) & y(0,0) & \ldots & y(2 m+1,0) & y(2 m, 0) \\
x(1,0) & x(0,0) & \ldots & x(2 m+1,0) & x(2 m, 0) \\
\cdot & \cdot & \ldots & \cdot & \cdot \\
y(1, m-2) & y(0, m-2) & \ldots & y(2 m+1, m-2) & y(2 m, m-2) \\
x(1, m-2) & x(0, m-2) & \ldots & x(2 m+1, m-2) & x(2 m, m-2)
\end{array}\right]_{(2 m-2) \times(2 m+2)}
$$

then $A \cdot(1 / t) B=J_{2 m+2}$, where $J_{2 m+2}$ is a diagonal matrix with $p p$ entry $(-1)^{p}$. Hence

$$
\text { column } \operatorname{rank}(A)=\text { row } \operatorname{rank}(A)=2 m+2 .
$$

Since $A$ has only $2 m-2$ columns, we have the desired contradiction. (This simple argument is due to Pat Rogers-see the proof of Theorem 3.10 in [2].)

We are now ready to define the relations $R_{n}$, and words $w_{n}$, inductively. To ease our notation, let $i$ and $j$ denote the standard enumerations of $\omega$ such that $i(n)<j(n) \leq n+1$ for all $n<\omega$ and $\{(i(n), j(n)): n<\omega\}=\{(r, s): r<s<\omega\}$. Define $w_{0}=\left[a_{2}, a_{3}\right]$ and $R_{0}=$ $\left[a_{0}, a_{1}\right]^{-1} w_{0}^{2}$; and for each $n<\omega$, pick $w_{n}$ such that $l\left(w_{n}^{t}\right)>2+n+\sum_{m<n} l\left(R_{m}\right)$ for all integers $t \neq 0$ - possible by the discussion above-and put $R_{n}=\left[a_{i(n)}, a_{j(n)}\right]^{-1} w_{n}^{2}$. As stated before, we let $K$ be the subgroup generated by $\left\{R_{n}: n<\omega\right\}$ and put $G=F / K$.

Property (a) of the lemma now follows from the definition of $G$. (Since $G$ is nil-2, it sufficed to arrange for the generators of $G^{\prime}$ to be squares.) To prove (b) choose $r<s$ such 
that $\left[a_{r}, a_{s}\right]$ does not appear in $\boldsymbol{R}_{\boldsymbol{m}}$ for $\boldsymbol{m}<\boldsymbol{n}$ and suppose $\left[a_{r}, a_{s}\right]$ were the square of some $w$, a product of $n$ commutators (modulo $K$ ). Then in $F,\left[a_{r}, a_{s}\right]^{-1} w^{2}=R_{0}^{k_{0}} R_{1}^{k_{1}} \ldots R_{t}^{k_{t}}$ for some integers $k_{0}, k_{1}, \ldots, k_{t}$, where $k_{t} \neq 0$. By the choice of $r$ and $s, t \geq n$. Recall that $R_{t}=\left[a_{i(t)}, a_{i(t)}\right]^{-1} w_{t}^{2}$; so $l\left(w_{t}^{2 k_{1}}\right) \leq 2+n+\sum_{m<t} l\left(R_{m}\right)$ which contradicts the choice of $w_{t}$.

Proof of the theorem. Let $G$ be as in the lemma; if $\sigma$ denotes $\forall x \exists y\left(x=y^{2}\right)$ then $G^{\prime} \vDash \sigma$. To construct $H$ (with $H^{\prime} \vDash \neg \sigma$ ) either take a non-trivial ultrapower of $G$, or use a simple compactness argument on

$T=\operatorname{Th}(G) \cup\{$ " $[c, d]$ is not the square of the product of $n$ commutators" $: n<\omega\}$,

where $c$ and $d$ are constants added to the language of groups. $T$ is consistent since $G$ can be interpreted as satisfying any finite subset of $T$, and $H$ can be any model of $T$.

\section{REFERENCES}

1. A. I. Mal'cev, Two remarks on nilpotent groups, Mat. Sb. (N.S.) 37(79) (1955), 567-572 (in Russian).

2. Pat Rogers, Preservation of saturation and stability in a variety of nilpoent groups, $J$. Symbolic Logic 46 (1981), 499-512.

L. P. D. van den Dries and Angus Macintyre

YALE UNIVERSTTY

New Haven, Connecticut, U.S.A.

A. M. W. Glass

Bowling Green State University

Bowling Green, Ohio, U.S.A.

Alan H. Mekler

UNIVERSITY OF WESTERn ONTARIO

London, Ontario, Canada

JOHN POLAND

Carleton University

Ottawa, Ontario, Canada

Current addresses:

JOHN POLAND

Simon Fraser UNIVERSITY

BURNABY 2

British Columbia, Canada

L. P. D. van den Dries

STANFORD UNIVERSITY

Palo Alto, California

U.S.A. 